\title{
Complicações da Oxigenação por Membrana Extracorpórea Venoarterial no tratamento terminal da insuficiência cardíaca
}

\author{
Complications of Venoarterial Extracorporeal Membrane Oxygenation in the terminal \\ treatment of heart failure
}

\section{Complicaciones de la Oxigenación por Membrana Extracorpórea Venoarterial en el tratamiento terminal de la insuficiencia cardíaca}

Ana Carolline Oliveira Torres ${ }^{1 *}$, Anna Carolyne Alves de Lima ${ }^{2}$, Fernanda Marinho de Souza ${ }^{3}$, Letícia Nogueira Carvalho Costa de Araújo', Liliane Rochemback ${ }^{4}$, Pedro Gabriel Matias Couras², Sara Oliveira Reis ${ }^{1}$, Sarah Menezes Gashti ${ }^{1}$, Tarsis Zaire Ferreira da Costa ${ }^{2}$, Glauco Kalil da Silva Pina ${ }^{5}$.

\section{RESUMO}

Objetivo: Identificar e descrever as complicações mais relevantes do suporte circulatório por meio da Oxigenação por Membrana Extracorpórea venoarterial (ECMO-VA) no tratamento terminal da Insuficiência Cardíaca (IC). Revisão bibliográfica: IC é uma síndrome clínica caracterizada por um déficit estrutural ou funcional, que compromete a funcionalidade ventricular, resultando em um aporte inadequado nas necessidades metabólicas tissulares. Desse modo, o uso do suporte de Oxigenação por Membrana Extracorpórea (ECMO) é indicado em cinco situações: ponte para recuperação, ponte para decisão, ponte para dispositivos de longa permanência, ponte para transplante e terapia de destino. Sendo assim, o suporte ECMO-VA é indicado para ofertar suporte cardíaco com função pulmonar conservada ou não. Além disso, durante a assistência do ECMO, ocorrem complicações secundárias ao tratamento que podem estar relacionadas ao quadro clínico do paciente ou ao dispositivo. Considerações finais: É notório que esse procedimento invasivo é uma das alternativas mais viáveis devido seus benefícios finais e resultados positivos na recuperação dos pacientes.

Palavras-chave: Cirurgia cardíaca, Procedimentos cirúrgicos cardiovasculares, Falência cardíaca, Oxigenação por membrana extracorpórea.

\begin{abstract}
Objective: To identify and describe the most relevant complications of circulatory support through Oxygenation by venoarterial Extracorporeal Membrane (ECMO-VA) in the terminal treatment of Heart Failure (HF). Bibliographic review: HF is a clinical syndrome characterized by a structural or functional deficit, which compromises ventricular functionality, resulting in an inadequate supply of metabolic tissue needs. Thus, the use of the Extracorporeal Membrane Oxygenation (ECMO) support is indicated in five situations: bridge for recovery, bridge for decision, bridge for long-term devices, bridge for transplant and destination therapy. Therefore, ECMO-VA support is indicated to offer cardiac support with preserved lung function or not. In addition, during ECMO assistance, complications secondary to treatment occur that may be related to the patient's clinical condition or the device. Final considerations: It is well known that this invasive procedure is one of the most viable alternatives due to its final benefits and positive results in the recovery of patients.
\end{abstract}

Key words: Thoracic surgery, Cardiovascular surgical, Heart failure, Extracorporeal membrane oxygenation.

\footnotetext{
${ }^{1}$ Universidade do Planalto Central Apparecido dos Santos (UNICEPLAC), Brasília - DF.

*E-mail: anacarollinetorres@gmail.com

2 Faculdade de Medicina Estácio de Juazeiro do Norte (Estácio - FMJ), Juazeiro do Norte - CE.

3 Universidade do Estado da Bahia (UNEB), Salvador - BA.

${ }^{4}$ Faculdade Estácio de Sá, Jaraguá do Sul - SC.

5 Universidade de Vassouras, Rio de Janeiro - RJ.
} 


\section{RESUMEN}

Objetivo: Identificar y descreer como complicaciones importantes del suporte circulatorio por el medio de Oxigenación por membrana Extracorpórea venoarterial (ECMO-VA) sin tratamiento terminal de Insuficiência Cardíaca (IC). Revisión bibliográfica: IC é uma síndrome clínica caracterizada por déficit estrutural o funcional, que compromete a funcionalidade ventricular, resultando em un aporte inadecuado nas necessidades metabólicas tisulares. Desse modo, o uso do suporte de Oxigenação por Membrana Extracorpórea (ECMO) é indicado en cinco situaciones: ponte para recuperação, ponte para decisão, ponte para dispositivos de longa permanência, ponte para transplante e terapia de destino. Sendo assim, o suporte ECMO-VA é indicado para ofertar suporteco cardíaco con función pulmonar conservada ou não. Além disso, durante una asistencia a ECMO, ocorrem complicações secundárias ao tratamento que podem estar relacionado ao quadro clínico do paciente ouo dispositivo. Considerações finais: É notório que esse procedimento invasivo é uma das alternativas mais viáveis devido seus benefícios finais e resultados positivos en la recuperación de dos pacientes.

Palabras clave: Cirugía del corazón, Procedimientos quirúrgicos cardiovasculares, Insuficiencia cardíaca, Oxigenación por membrana extracorpórea.

\section{INTRODUÇÃO}

A insuficiência cardíaca (IC) com fração de ejeção reduzida é uma patologia caracterizada por déficit na capacidade sistólica do coração. Desse modo, os tecidos periféricos começam a se manter em um estado de déficit circulatório. Em alguns casos, o corpo pode entrar em colapso e evoluir para um choque cardiogênico ou até mesmo para uma parada cardiorrespiratória (DANGERS L, et al., 2017).

Diante disso, surge a necessidade da utilização de estratégias para estabelecer o suporte hemodinâmico em pacientes críticos. Tendo em vista esse quadro, em 1954 foi usado pela primeira vez um dispositivo de Oxigenação por Membrana Extracorpórea (ECMO), em primeiro caso, dentro de um procedimento de cirurgia cardíaca. Desde então, essa técnica tem sido aprimorada e vem sendo alvo de estudos e pesquisas em todo o mundo (CHAVES RCF, et al., 2019).

A ECMO constitui-se de um dispositivo mecânico de circulação extracorporal, que vem sendo amplamente utilizado como terapia de resgate em pacientes graves com comprometimento hemodinâmico, acompanhado ou não de falência respiratória, em situações clínicas potencialmente reversíveis e refratárias ao tratamento convencional, até que a terapia definitiva seja instalada (GUGLIN M, et al., 2019).

Existem duas modalidades desse dispositivo: a venovenosa (ECMO-VV), que substitui os pulmões deficientes, mas com função cardíaca preservada e a venoarterial (ECMO-VA), que fornece suporte respiratório e hemodinâmico. A ECMO-VA funciona por meio de um sistema de cânulas inseridas em acessos centrais venosos e arteriais, drenando e bombeando todo o sangue do paciente, para que haja as trocas gasosas necessárias para oxigenação sanguínea eficaz e suporte hemodinâmico controlado. Neste cenário, é imprescindível a heparinização plena, a fim de evitar uma trombogênese maciça, que pode ocorrer devido ao contato sanguíneo com as cânulas (CHAVES RCF, et al., 2019).

Embora não existam indicações precisas do uso da ECMO-VA dentro da diretriz de insuficiência cardíaca proposta pela Sociedade Brasileira de Cardiologia (SBC), a aplicação dessa ferramenta torna-se uma importante medida de suporte circulatório de curto prazo. Destaca-se sua utilização nos quadros de complicações da insuficiência cardíaca (choque cardiogênico e parada cardiorrespiratória), principalmente nos casos em fases terminais, nos que aguardam um suporte mecânico de longo prazo (Dispositivo de Assistência Ventricular [DAV]) e nos que o transplante cardíaco é a solução definitiva, de modo que desempenha papel essencial na estabilização hemodinâmica e perfusão tecidual periférica (ROHDE LE, et al.,2018; LIMA E, et al. 2015).

Contudo, esse suporte circulatório apresenta um grau avançado de complexidade, demandando indicações restritas e uma equipe multidisciplinar preparada e treinada, composta por médicos, enfermeiros 
e fisioterapeutas aptos para desempenhar tal função. Por isso, o custo da ECMO pode ser elevado, mas tendo em vista o benefício final, o gasto ofertado torna-se uma alternativa viável (ROMANO T, et al., 2017; ABRAMS D, et al., 2018).

Ademais, apesar dos evidentes benefícios da ECMO-VA como suporte temporário e como prevenção da progressão da falência cardíaca, as complicações relacionadas à ECMO podem provocar desfechos desfavoráveis, sendo, portanto, um fator preponderante na tomada de decisões para instauração dessa modalidade terapêutica (NAKASATO GR, et al., 2020).

Essas complicações podem ser divididas em 3 grandes grupos: relacionadas ao quadro clínico, à anticoagulação e ao dispositivo. Dentre as complicações relacionadas ao quadro clínico dos pacientes, as complicações mais prevalentes são: renais (38-75\%); neurológicas (5,9-21\%); infecciosas (11-33\%) e vasculares (3,9-12\%) (MIANA L, et al., 2018).

Já naquelas relacionadas à anticoagulação, é muito comum encontrar queda da hemoglobina por hemodiluição e consumo de fatores de coagulação. Em relação ao dispositivo, ocorrem relatos de falha mecânica, ruptura ou desconexão dos tubos e falha na oxigenação por problemas relacionados com a membrana, sendo esses fatores decorrentes exclusivamente de falha funcional e estrutural da ECMO (LEHLE K, et al., 2017).

No que concerne à decisão do uso da ECMO-VA, cabe, ainda, considerar os preditores que podem elevar as chances de os pacientes desenvolverem alguns tipos de complicações. Dentre os diversos preditores, existem alguns mais prevalentes, como tempo na UTI, tempo de uso da ECMO, patologia de base, idade do paciente, creatinina elevada, infecção associada e baixa da hemoglobina (AYYILDIZ P, et al., 2017).

Diante do exposto, o objetivo desta revisão é evidenciar as complicações mais relevantes da instauração do suporte circulatório por meio da ECMO-VA, no tratamento terminal da insuficiência cardíaca, que resulta ou em choque cardiogênico ou em parada cardiorrespiratória.

\section{REVISÃO BIBLIOGRÁFICA}

\section{Insuficiência Cardíaca}

A Insuficiência Cardíaca (IC) é a síndrome clínica resultante de alterações cardíacas estruturais ou funcionais, as quais dificultam a ação ventricular de preenchimento e de ejeção sanguínea. Com isso, o bombeamento cardíaco torna-se deficitário no atendimento de necessidades metabólicas tissulares devido à redução no débito cardíaco e/ou das elevadas pressões de enchimento no repouso ou no esforço. Logo, tal síndrome é vista como a via final comum da maioria das cardiopatias (SANTOS IS e BITTENCOURT MS, 2008).

Dessa maneira, a terapêutica e o prognóstico baseiam-se no perfil clínico-hemodinâmico e no estadiamento da doença. O perfil clínico-hemodinâmico define as condições de volemia e de perfusão, por meio da avaliação de sinais e sintomas de hipervolemia/hipovolemia e de baixa perfusão periférica. Os pacientes com sintomas e sinais clínicos de congestão são classificados como congestos; na ausência destes, como secos; apresentando sinais de baixo débito, são denominados frios; e com perfusão periférica preservada, como quentes. Logo, há quatro cenários possíveis: a) Pacientes congestos sem baixo débito: quente-congesto; b) Pacientes congestos com baixo débito: frio-congesto; c) Pacientes sem congestão com baixo débito: frio-seco; d) Pacientes sem sinais de congestão ou de baixo débito: quente-seco (MANGINI S, et al., 2013).

Já o estadiamento patológico é fundamentado em três critérios: 1. A fração de ejeção; 2 . Gravidade dos sintomas; 3. Progressão da doença. A fração de ejeção (FE) é avaliada por meio da função sistólica, que é calculada através da relação entre o volume diastólico final e o volume de ejeção do ventrículo durante a sístole, sendo a FE considerada adequada quando está entre 50-70\% e reduzida quando está abaixo de $40 \%$. Há também a classificação funcional da New York Heart Association (NYHA), a qual avalia limitações sintomáticas do paciente para desempenho de atividades cotidianas. Por fim, a escala de estágio da IC 
desenvolvida pela American Heart Association (AHA) avalia a função cardíaca a partir da progressão estrutural de lesão cardíaca associada aos sintomas (ROHDE LEP, et al., 2018; FREITAS AK e CIRINO, RHD, 2017).

Logo, a IC é considerada uma doença progressiva, pois, independente da regressão sintomática, a qual pode ser estabelecida pelo tratamento clínico, as disfunções estruturais, muitas vezes, continuam progredindo. Assim, a evolução patológica pode vir a cursar com o choque cardiogênico quando manifestados sinais de hipoperfusão e sinais de congestão, ou seja, quando o paciente se apresenta frio-congesto, determinando, assim, uma Insuficiência Cardíaca Descompensada (SANTOS IS e BITTENCOURT MS, 2008). Desse modo, conclui-se que quando o choque cardiogênico não é responsivo ao tratamento clínico, pode ser considerada a escolha de dispositivos de assistência ventricular (DAV) como estratégia terapêutica. Por sua vez, estes dispositivos podem ser divididos como de curta, média ou longa duração. Dentre os de curta duração, inclui-se a ECMO, suporte circulatório mecânico, principal foco deste artigo (MANGINI S, et al., 2013).

\section{Oxigenação por Membrana Extracorpórea (ECMO)}

Mesmo sob administração de fármacos inotrópicos, diuréticos e vasopressores, caso o choque cardiogênico evolua com débito cardíaco reduzido e insuficiente, este passa a ser definido como choque cardiogênico refratário. Diante disso, as causas de choque cardiogênico são: miocardite fulminante, infarto do miocárdio, exacerbação de insuficiência cardíaca crônica, arritmias intratáveis, intoxicação por drogas, insuficiência cardíaca pós cardiomiopatia, entre outras. Com isso, a assistência circulatória tem como objetivo o resgate hemodinâmico e a tentativa de restabelecimento da função cardíaca, a fim de possibilitar o tratamento adequado e reduzir mortes (NAKAHIRA ES e GALAS FRBG, 2016).

Nessa perspectiva, sabe-se que, desde o início da sua aplicação até os dias atuais, o procedimento da ECMO passou por aperfeiçoamentos expressivos na execução do dispositivo, na condução do paciente e, portanto, nas conclusões dos tratamentos. Dessa forma, tornou-se, atualmente, um dos mecanismos predominantes de suporte de vida extracorpóreo (CHAVES RCF, et al., 2019; KATTAN J, et al., 2017).

No que tange à assistência circulatória mecânica, a assistência de curta duração é a que possui o uso, em geral, menor que 30 dias. Destarte, o suporte circulatório temporário é indicado em cinco situações: ponte para recuperação; ponte para decisão; ponte para dispositivos de longa permanência; ponte para transplante e terapia de destino (NAKAHIRA ES e GALAS FRBG, 2016).

Além das indicações anteriores, a ECMO apresenta duas modalidades de uso: venovenosa e venoatrial. A modalidade ECMO venovenosa (ECMO-VV) é escolhida no cenário de insuficiência respiratória com função cardíaca conservada. A modalidade ECMO venoarterial (ECMO-VA) é recomendada para ofertar suporte cardíaco com função pulmonar conservada ou não (CHAVES RCF, et al., 2019).

Ainda no que tange à ECMO-VA, constata-se que a mesma necessita de dois tipos de cânula, uma de drenagem, que é incorporada em um acesso venoso, e uma de retorno, que é incorporada em um acesso arterial. Além disso, esse tipo de ECMO possui duas formas de classificação: central ou periférica. $\mathrm{Na}$ classificação central, o átrio direito é inserido, de modo direto, pela cânula de drenagem e o segmento ascendente da artéria aorta é inserido, de modo direto, pela cânula de retorno. Já na configuração periférica, a drenagem é realizada pelas veias jugular ou femoral e o retorno é realizado pelas artérias carótidas, femoral ou axilar. Após explicado esse mecanismo, entende-se como a ECMO-VA oferece suporte circulatório de modo a eliminar a circulação pulmonar (GUGLIN M, et al., 2019).

Nesse viés, a ECMO tem por objetivo manter a volemia e a perfusão tissular adequadas de órgãos acometidos e, para tanto, necessita de um acesso vascular, o qual pode ser executado por intermédio de uma via percutânea ou via cirúrgica. Este acesso deve possuir um cateter ou uma cânula com bombas mecânicas (NAKASATO GR, et al., 2020; GUGLIN M, et al., 2019).

Esse procedimento é utilizado como terapia de resgate de primeira linha em pacientes com choque cardiogênico refratário e consiste em um sistema composto por um cateter venoso percutâneo, tratado com 
heparina. Então, o sangue do paciente é drenado e mandado para uma bomba de propulsão, a qual impulsiona o sangue do paciente para a membrana oxigenadora, onde ocorre a disseminação dos gases entre o sangue do paciente e a corrente de gás fresco, ou seja, o oxigênio e o gás carbônico são trocados. Dessa forma, o sangue venoso torna-se oxigenado e é devolvido para o paciente por meio de um sistema venoso ou arterial (CHAVES RCF, et al., 2019).

Depreende-se, então, que a ECMO venoarterial (ECMO-VA) é atualmente a escolha para assistência circulatória, já que não depende do débito cardíaco do paciente e é utilizada em situações clínicas que têm potencial de ser revertidas. Além de não depender do débito cardíaco do paciente, a ECMO pode ser utilizada em casos de emergência, possui fácil e rápida canulação e necessita apenas de anestesia local para a sua efetivação. Assim, a ECMO-VA oferece uma melhora rápida do estado circulatório e aumento significativo na perfusão do tecido. Desse modo, esse procedimento influencia significativamente na prática atual do manejo de pacientes frios-congestos. No entanto, por ser um método invasivo, necessita de anticoagulação e pode ser associado a possíveis complicações (NAKASATO GR, et al., 2020; NAKAHIRA ES e GALAS FRBG, 2016).

\section{Complicações decorrentes da ECMO}

Durante o suporte extracorpóreo, ocorrem complicações secundárias ao tratamento, as quais podem estar relacionadas ao quadro clínico do paciente, à anticoagulação ou ao dispositivo. Considerando as possíveis complicações relacionadas ao quadro clínico do paciente, observam-se as complicações renais, hemorrágicas, infecciosas e neurológicas (NAKASATO GR, et al., 2020).

Referente às complicações de anticoagulação, estas são associadas à elevação do volume de plasma sanguíneo em relação aos glóbulos vermelhos, resultando na diminuição da concentração desses glóbulos na circulação e ao consumo de fatores de coagulação, bem como o baixo nível de plaquetas (trombocitopenia) induzido pela heparina. A respeito das complicações relacionadas ao dispositivo, sabe-se que as mesmas acontecem em até $11 \%$ dos pacientes e tem-se como relevante as falhas mecânicas, sendo elas: a falha na membrana oxigenadora ou na bomba, a troca do circuito ou da membrana, a ruptura ou desconexão da tubulação e entrada de ar no sistema, motivo que leva à embolia gasosa (CHAVES RCF, et al., 2019).

No que concerne às complicações renais, pouco é sabido em relação à fisiopatologia da Lesão Renal Aguda (LRA) em pacientes submetidos à ECMO, entretanto, sabe-se que diversas são as causas que podem levar à LRA, dentre elas estão às condições clínicas do paciente pré-ECMO, o mecanismo do dispositivo propriamente dito, o tempo de internação na Unidade de Terapia Intensiva (UTI) e a hemólise, causada pela diminuição da perfusão tecidual. Por isso, como medida preventiva para a LRA, é importante que se monitore a pressão do sistema, com o fito de manter um fluxo ideal e evitar que a pressão venosa seja muito negativa (CHAVES RCF, et al., 2019; LEHLE K, et al., 2017).

Quanto às complicações hemorrágicas, estas são responsáveis por uma mortalidade significativa (40 a $60 \%$ ) e estão associadas à intensidade de anticoagulação, visto que o Tempo de Tromboplastina Parcial Ativada e o nível de plaquetas foram encontrados como preditores. Isso ocorre, pois durante a ECMO há o consumo de plaquetas e de fatores de coagulação devido ao contato do sangue com uma superfície não endotelial, necessitando de anticoagulação com o intuito de prevenir trombose no circuito. Logo, é fulcral que a equipe multidisciplinar estabeleça um nível ideal de anticoagulação, evitando as complicações secundárias. Assim, o ácido tranexâmico e o fator VIlla de coagulação são utilizados para diminuir as desordens hemorrágicas, visto que estes impedem o sangramento em 34\% dos casos (NAKASATO GR, et al., 2020; RATNANI I, et al., 2018).

Ainda na conjuntura das complicações, observam-se causas infecciosas, sendo estas associadas ao tempo em ECMO, quando o paciente permanece mais do que $48 \mathrm{~h}$ em suporte, bem como ao nível sérico de creatinina elevado e à imunossupressão, devido ao comprometimento do sistema imune. Além disso, os pacientes em ECMO possuem risco aumentado de infecções devido à canulação dos vasos, fator esse que permite a entrada de agentes infecciosos. Dentre as infecções, as mais prevalentes são as de corrente sanguínea, sendo os patógenos gram-negativos os mais preponderantes (AYYILDIZ P, et al., 2017). 
Sobre as complicações neurológicas, considera-se o nível elevado de ácido lático pré-ECMO (>10 $\mathrm{mmol} / \mathrm{L}$ ), os baixos níveis de hemoglobina, a trombocitopenia e a função renal prejudicada como preditores desse tipo de comprometimento. Para tal, deve-se realizar um reparo intensivo e aperfeiçoado desses parâmetros para que se obtenha uma menor ocorrência de acidente vascular isquêmico e de mortalidade (MIANA L, et al., 2018).

Findando os fatores de complicação relacionados ao quadro clínico do paciente, existem outras desordens que devem ser citadas, entre elas: a trombocitopenia grave, a linfocele e os fenômenos tromboembólicos. A trombocitopenia grave, a qual aumenta o risco de hemorragia, tem a baixa contagem de plaquetas após a canulação como preditor e também pode ser explicada pela gravidade inicial da doença crítica e pelo desenvolvimento da falência de múltiplos órgãos durante a terapia com a ECMO. A linfocele é caracterizada pelo acúmulo de linfa em qualquer região do corpo, o que normalmente é causado por lesão de vasos que conduzem este líquido, é específica dos casos de inserção de canulação femoral, é grave e requer, em alguns casos, intervenção cirúrgica (NAKASATO GR, et al., 2020).

Em relação aos fenômenos tromboembólicos como a Trombose Venosa Profunda (TVP) e Tromboembolismo Pulmonar (TEP), estão associados às áreas de baixo fluxo causadas pelo enrijecimento do vaso, decorrente da coagulopatia e são motivados pelo contato do vaso com uma superfície não endotelizada. Além disso, sabe-se que pacientes submetidos ao procedimento de ECMO mediante canulação femoral apresentam incidência de complicações vasculares em 17 a 35\% dos casos (TRUDZINSKI FC,et al., 2016). Nesse âmbito, o consenso da Extracorporeal Life Support Organization (ELSO) define que, mesmo com as prováveis complicações decorrentes da ECMO, não são verificadas contraindicações absolutas para a sua indicação. No entanto, os riscos e os benefícios do procedimento devem ser estudados de forma particular a cada paciente. Para tanto, demonstra-se que as indicações mais relevantes da ECMO-VA são o choque cardiogênico, as cardiomiopatias, cardiopatias congênitas e a miocardite. Já as contraindicações relativas são a hemorragia ativa não controlada, a falência cardíaca irreversível e a neoplasia sem perspectiva de tratamento (CHAVES RCF, et al., 2019).

\section{Equipe Multidisciplinar}

A ECMO-VA deve ser executada o mais rápido possível e necessita do suporte de uma equipe multidisciplinar. Esta é composta por diversos profissionais, sendo eles, geralmente, os cirurgiões cardiotorácicos, os perfusionistas, os intensivistas, os anestesiologistas e os profissionais de cuidados respiratórios. Dessa forma, cabe a esses profissionais a realização de protocolos sedativos e analgesia, a titulação de drogas vasoativas, o ajuste da anticoagulação norteada por metas específicas, além da coleta de exames laboratoriais e da inspeção frequente do circuito por meio da inspeção de áreas escurecidas ou esbranquiçadas e, caso sejam percebidos esses aspectos, haverá sugestão de coagulação do sistema (ABRAMS D, et al., 2018).

Nesse contexto, para uma implantação bem-sucedida, torna-se fulcral que a equipe multidisciplinar, de forma a assegurar os cuidados dos pacientes submetidos à ECMO, conheça a fisiologia e as possíveis complicações decorrentes do procedimento, de modo a planejar, de forma individualizada, o cuidado. Para tanto, é crucial que sejam realizados programas de educação e treinamento específicos sobre a ECMO para que a equipe adquira os conhecimentos necessários para prestar um atendimento ideal às necessidades dos pacientes. Com base nisso, há uma redução expressiva no número de mortes em Unidades de Terapia Intensiva (UTIs) e na ocorrência de problemas com a canulação e com os eventos cardiovasculares (NAKASATO GR, et al., 2020). Dessa maneira, a equipe multidisciplinar ofertará um cuidado especial a cada paciente em ECMO, bem como o apoio psicossocial aos familiares, com vistas a otimizar o uso da metodologia e alcançar melhores resultados (CHAVES RCF, et al., 2019).

\section{CONSIDERAÇÕES FINAIS}

Verificou-se nesse trabalho a relevância da ECMO-VA para assistência circulatória, a qual é indicada em casos de insuficiência cardíaca terminal e suas complicações (choque cardiogênico e parada 
cardiorrespiratória) e tem mostrado um resultado satisfatório, entre eles: o resgate hemodinâmico, aumento da volemia e aumento da perfusão tecidual. Ainda que o suporte extracorpóreo possa causar complicações renais, hemorrágicas, infecciosas, neurológicas e complicações relacionadas à anticoagulação, esse procedimento invasivo é uma das alternativas mais viáveis devido seus benefícios finais e resultados positivos na recuperação dos pacientes. Portanto, para que isso ocorra, necessita-se de uma equipe multidisciplinar treinada e capacitada.

\section{REFERÊNCIAS}

1. ABRAMS D, et al. Position paper for the organization of ECMO programs for cardiac failure in adults. Intensive Care Med, 2018; 44: 717-129.

2. ARAÚJO I, et al. Ativação de Oxigenação por Membrana Extracorpórea: uma atitude terapêutica a se ponderar. Rev Bras Ter Intensiva, 2019;31(3):282-288.

3. AYYILDIZ P, et al. Avaliação de infecções hospitalares em pacientes pediátricos com suporte de oxigenação por membrana extracorpórea. Jornal Brasileiro de Cirurgia Cardiovascular, 2017;32(6):468-474.

4. CHAVES RCF, et al. Oxigenação por Membrana Extracorpórea: revisão da literatura, São Paulo. Revista Brasileira de Terapia Intensiva, 2019; 31(3): 410-424.

5. COLAFRANCESCHI AS, et al. Assistência Circulatória com Oxigenação por Membrana Extracorpórea (ECMO) no Adulto: um conceito Falido ou Esquecido?. Arquivos Brasileiros de Cardiologia, 2008;91(1):36-41.

6. ROHDE LE, et al. Diretriz Brasileira de Insuficiência Cardíaca Crônica e Aguda, Arquivos brasileiros de cardiologia, 2018; 111(3): 436-539.

7. DANGERS L, et al. Extracorporeal Membrane Oxygenation for Acute Decompensated Heart Failure. Critical Care Medicine, 2017; 45(8): 1359-1366.

8. FREITAS AKE, CIRINO RHD. Manejo ambulatorial da insuficiência cardíaca crônica. Rev Med UFPR [Internet]. 2017;4(3):123-36.

9. GUGLIN M, et al. Venoarterial ECMO for Adults. Journal of the American College of Cardiology, 2019; 73(6):698-716.

10. JASSERON C, et al. Impact of heart transplantation on Survival in Patients on Venoarterial Extracorporeal Membrane Oxygenation at Listing in France. Transplantation, 2016; 100(9): 1979-1987.

11. KATTAN J, et al. Oxigenação por membrana extracorpórea neonatal e pediátrica em países emergentes da América Latina. Jornal de Pediatria, 2017; 93(2): 120-129, 2017.

12. LEHLE K, et al. Prevalence of hemolysis and metabolic acidosis in patients with circulatory failure supported with extracorporeal life support: a marker for survival?. European Journal of Heart Failure Supplements, 2017; 19(2): 110116.

13. LIMA E, et al. Experiência na Utilização de ECMO na Disfunção Primária de Enxerto Pós-Transplante Cardíaco Ortotópico, Brasília. Arquivos Brasileiros de Cardiologia, 2015; 105 (3): 285-291.

14. MANGINI S, et al. Insuficiência cardíaca descompensada. Einstein (São Paulo), São Paulo. 2013; 11: 383-391.

15. MANGINI S, et al. Transplante Cardíaco: revisão, São Paula. Einstein, 2015; 13(2):310-8.

16. MANN DL, et al. Braunwald's heart disease: a textbook of cardiovascular medicine. 10th ed. Philadelphia: Elsevier, 2015.

17. MIANA L, et al. Rational Use of Mechanical Circulatory Support as a Bridge to Pediatric and Congenital Heart Transplantation. Jornal Brasileiro de Cirurgia Cardiovascular, 2018; 33(3): 242-249.

18. NAKAHIRA ES e GALAS FRBG. Utilização de oxigenação por membrana extracorpórea (ECMO) no choque cardiogênico refratário: relato de caso e revisão da literatura, São Paulo. Revista de Medicina, 2016; 95(4):168-74.

19. NAKASATO GR, et al. Preditores de complicações da oxigenação por membrana extracorpórea, São Paulo. Revista Brasileira de Enfermagem, 2020; 73(2): e20180666.

20. NOMENCLATURE AND CRITERIA FOR DIAGNOSIS OF DISEASES OF HEART AND GREAT VESSELS. The Criteria Committee of the New York Heart Association. 9th ed. Boston: Little Brown, 1994.

21. RATNANI I, et al. The Role and Impact of Extracorporeal Membrane Oxygenation in Critical Care. Methodist Debakey Cardiovasc J, 2018; 14(2): 110-119

22. ROHDE LEP, et al. Diretriz Brasileira de Insuficiência Cardíaca Crônica e Aguda. Arq. Bras. Cardiol., 2018; 111(3) 436-539.

23. ROMANO T, et al. Suporte respiratório extracorpóreo em pacientes adultos, São Paulo. Jornal Brasileiro de Pneumologia, 2017; 43(1): 60-70

24. SAFFARZEDEH A, BONDE P. Options for temporary Mechanical circulatory support.Journal of Thoracic Disease, 2015; 7(12): 2102-2111.

25. SANTOS IS, BITTENCOURT MS. Insuficiência cardíaca. Revista de Medicina, 2008; 87 (4): 224-231.

26. SANTOS SE. Desfechos clínicos de pacientes pediátricos tratados com oxigenação por membrana extracorpórea. Acta Paulista de Enfermagem, 2016; 29(4): 405-412.

27. TRUDZINSKI FC, et al. Runtime and aPTT predict venous thrombosis and thromboembolism in patients on extracorporeal membrane oxygenation: a retrospective analysis. Annals of intensive care, 2016; 6(1): p. 1-8.

28. YANCY CW, et al. ACCF/AHA guideline for the management of heart failure: a report of the American College of Cardiology Foundation/American Heart Association Task Force on practice guidelines. Circulation, 2013. (16): 2014327. 